\title{
Juxta-articular adiposis dolorosa: What is it? Report of 2 cases
}

\author{
J. EISMAN AND R. L. SWEZEY \\ From the Division of Rheumatology and Rehabilitation Medicine, University of California, Los Angeles, USA
}

SUMMARY Juxta-articular adiposis dolorosa may be defined as the localised accumulation of painful fat near joints, most commonly the knee. It exists as a separate entity that must be distinguished from other causes of knee pain. We present 2 patients with this condition and review the literature. Various approaches to therapy are discussed. A classification of painful fat near joints is proposed.

Juxta-articular adiposis dolorosa (JAD)-does it exist? What is it? Literally it means painful fat near a joint. We believe the condition does exist as a separate entity and that it may coexist with other disorders affecting joints. We describe 2 patients with this disorder and review the available literature. It is important to recognise the condition as a not so rare cause of knee pain. Nevertheless we have been unable to find a single publication about this disorder in the rheumatology journals and reviews. A classification of painful fat in and around joints is proposed, and various pharmacological and physiotherapeutic measures are discussed.

\section{Case reports}

\section{CASE 1}

This patient is a 50-year-old Caucasian woman who presented with a 2-year-old history of bilateral knee pain associated with the development of tender fatty growths on the medial aspects of both knees. Later these masses became associated with painful paraesthesiae travelling down the anteromedial aspects of both calves. The knee joints did not swell. The patient had always been mildly obese. She was not menopausal. No other abnormalities were detected.

Physical examination revealed a mildly obese woman. She was normotensive. The only abnormalities were confined to her knees which revealed moderate valgus deformities bilaterally. Both knees had full range of motion, minimal crepitation, mild instability of the medial collateral ligaments, and no

\section{Accepted for publication 22 August 1978}

Correspondence to R. L. Swezey, MD, Arthritis and Back Pain Center, 2200 Santa Monica Blvd., Santa Monica, California 90404, USA. effusion. Just medial to each knee was a fist-sized mass of rather tense and extremely tender fatty tissue. There was also tenderness of both pes anserine bursae.

Investigations revealed a normal complete blood count, differential white cell count, and erythrocyte sedimentation rate. Biochemical tests were normal except for a serum calcium of $11.2 \mathrm{mg} / \mathrm{dl}$, serum phosphorus of $2 \cdot 3 \mathrm{mg} / \mathrm{dl}$, and a serum parathyroid hormone level of $109 \mu 1 \mathrm{Eq} / \mathrm{ml}$ (normal 90-100) (at the time of writing no disposition of her hyperparathyroidism has been made). Thyroid function tests, glucose tolerance test, RA latex, ANA, serum uric acid, and an electromyogram of both lower limits were normal or negative. Roentgenograms (including weight-bearing views) of both knees were also normal. A fat biopsy from the mass on the left knee was also normal.

A diagnosis of JAD was made. She was treated with aspirin, naproxen, indomethacin, and ibuprofen in full anti-inflammatory doses. None of the nonsteroidal agents relieved her pain. A course of carbamazepine (given elsewhere) was also without benefit. Local injection (of the painful fat) with lignocaine exacerbated her symptoms. Injection of the left anserine bursa with triamcinolone acetonide suspension and lignocaine was ineffective, and attempts at weight reduction were also unsuccessful. A trial of corrective shoes with medially wedged soles and heels in an effort to minimise valgus angulation and medial knee contact gave no relief. Finally, intermittent pneumatic compression (Jobst) was used in an attempt to compress the fat and possibly mobilise suspected local oedematous accumulations in fatty tissue. The patient was able to tolerate $65 \mathrm{mmHg}$ of pressure for 1 hour twice daily, but when the pressure was increased to $70 \mathrm{mmHg}$ 
she felt marked discomfort and the treatment had to be abandoned. Elastic support hose was also poorly tolerated and ineffective in relieving the patient's discomfort.

\section{CASE 2}

This patient is a 54-year-old Caucasian woman who was initially seen for seropositive nodular rheumatoid arthritis. During the last 20 years she had gained $75 \mathrm{lb}(34 \mathrm{~kg})$ in weight. Fifteen years previously she had noticed the fat pads on the medial aspects of her knees became enlarged. On examination the fat pads were tender to palpation, and this tenderness was more severe and appeared to be distinct from that elicited on deep palpation of the medial joint margins. Neither knee had an effusion. She also had active synovitis in her wrists, metacarpophalangeal and proximal interphalangeal joints, ankles, and metatarsophalangeal joints. Roentgenograms of her knees revealed only osteophytes on the intercondylar notches. No biopsy was performed. A diagnosis of JAD was made in addition to rheumatoid arthritis. Fig. 1 illustrates bilateral medial fat pad hypertrophy in this patient.

\section{Discussion}

The topic of painful fat in and around joints and in particular the knee has been made very confusing by the various names used to describe the same and different clinicopathological entities. In addition, most of the reports are old and the patients were not investigated by present-day methods. An attempt to classify these disorders on an anatomicopathological basis is given in Table 1 .

The conditions affecting the fatty tissue around joints, most commonly the knee, have been called by a variety of names. Their relationship to each other

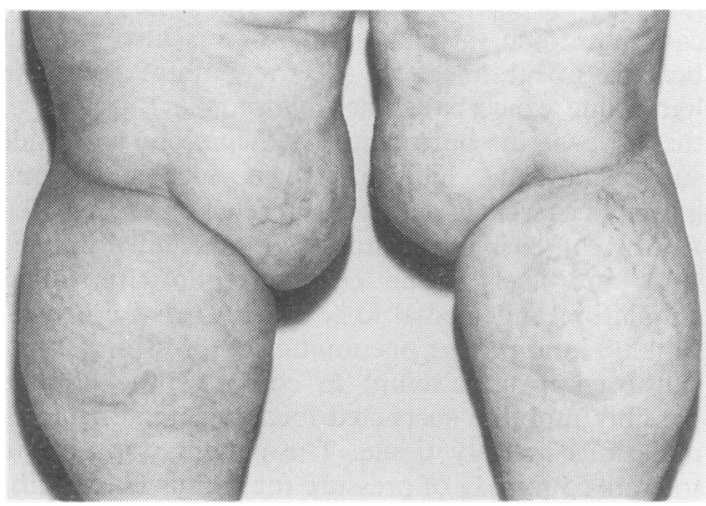

Fig. 1 Case 2. Bilateral medial fat pad hypertrophy
Table 1 Classification of painful fat around joints

\begin{tabular}{l} 
A. Extra-articular: \\
(1) Juxta-articular adiposis dolorosa (Also known as (a) pan \\
niculitis, (b) Dercum's syndrome) \\
B. Intra-articular: \\
(1) Hoffa's disease \\
(2) Premenstrual water retention syndrome \\
C. Fat legs and orthostatic oedema (lipoedema) \\
\hline
\end{tabular}

and to Dercum's disease is unclear. Indeed, they may $\vec{\circ}$ represent a spectrum of the same disease expressingw different levels of severity and associated systemict phenomena.

What Kling calls 'juxta-articular adiposis dolorosa $\dot{\omega}_{\infty}$ (Kling, 1937), Gram terms 'symptom triad of their post-climactic period-adipositas dolorosa - arthritis $₹$ genuum-hypertensio arterialis' (Gram, 1930), ando Smillie cells 'panniculitis-the fibro-fatty syndrome'? (Smillie, 1974c) are probably the same disease and best called 'juxta-articular adiposis dolorosa'. Kling reported on 112 cases (Kling, 1937). He observedo accumulations of tender subcutaneous fat aroundळ joints. It occurred most frequently in obese women past middle age and was usually bilateral. It mostc commonly affected the knees, less frequently the elbows, and rarely the ankles and hips. The patientso complained of pain, stiffness, or crepitus. Some hads paraesthesiae. Many had menopausal symptoms and systolic hypertension, as would be expected in this age group. These patients often had depressed $Q$ arches, varicose veins, acroparaesthesiae and vaso- $\overrightarrow{\overrightarrow{0}}$ motor disturbances. Histologically, normal fat was found. Kling, feeling that this was an early stage of Dercum's syndrome, thought the evolution was as. follows: initially in Dercum's syndrome there is a monoarticular (usually knee) hypersensitivity of subcutaneous masses of fat, then a successive involvement of other joints, and finally of the torso. The joint changes as such are minimal.

Gram described the frequent occurrence of obesityo with tender subcutaneous infiltrations, 'deforming? arthritis' of the knee, and arterial hypertension in women around and after the climacteric age (Gram) 1930). It was commoner in multiparous women. The patients complained of obesity, dyspnoea, and palpitations. They often had vague shooting pains anywhere in their bodies. Some complained of 'rheumatic knees' with creaking and stiffness. Physical examination revealed multiple tender areast in subcutaneous regions. These areas were distincts infiltrations. $X$-rays often showed 'arthritis defor 0 mans' with osteophytes at the joint margins and intercondylar notches. He stated that Dercum's巴 syndrome covers only the extreme cases.

Smillie reports that a common bilateral finding in women after the menopause is an ill defined fibrofatty 
mass situated over the anteromedial aspect of the medial tibial condyle (Smillie, 1974c). The swelling may occur at any age, however. The mass is sometimes tender, and a peau d'orange effect may be felt when the mass is grasped between the thumb and finger. Smillie stresses that this disorder must be distinguished from pes anserine bursitis. He does not advise surgery and warns that a unilateral procedure leaves a poor cosmetic result. This appears to be the least severe end of the spectrum and similar to what both our patients suffer. We prefer to call this group of diseases 'JAD'.

What Allen and Hines called lipoedema of the legs may be yet another variant of this disorder (Allen and Hines, 1940). Lipoedema (a syndrome characterised by fat legs and orthostatic oedema) affected only women (in their series). The patients had a long history of swollen legs, particularly below the knees; the swelling became worse when they were erect and during warm weather. Physical examination revealed obesity of the legs and buttocks. The oedema did not involve the feet. The fatty tissues were usually tender. Treatment with diuretics did not help. Heat and massage offered only temporary relief. They found elastic stockings were often not tolerated. Forty additional patients with 'lipoedema' were studied (Stallworth et al., 1974). Normal fat was found on biopsy. Most had normal phlebograms and lymphangiograms. Stallworth et al. stated that the tenderness was localised to the subcutaneous tissue just below the knee, where a bulge of fat was prominent. They found compression bandages applied progressively more tightly at weekly intervals relieved most of their patients' symptoms. The pain and tenderness recurred when the bandages were removed. The patients thus required permanent support from knee to toe. Low fat diet, dependent drainage, and diuretics were of no help.

Dercum first described the syndrome which now bears his name (Dercum, 1892). The cardinal features have been listed by Blomstrand et al., (1971) as: (1) Painful, circumscribed or diffuse, fatty deposits, often localised to the lower legs; (2) generalised obesity of women (it does occur in men), usually postmenopausal; (3) asthenia, weakness, and marked fatiguability; (4) psychic phenomena, including emotional instability, melancholia, epilepsy mental confusion, and true dementia.

The early reports of Dercum's syndrome noted variable pathology in endocrine glands at necropsy (Dercum, 1892; Dercum and McCarthy, 1902; Price, 1909; Winkelman and Eckel, 1925). The evidence for a pluriglandular involvement was later doubted (Steiger et al., 1952). The same authors also found a course of ACTH was of no benefit in treatment (Steiger et al., 1952). Two patients with
Dercum's syndrome were studied extensively (Blomstrand et al., 1971). Both gave normal results to tests of thyroid, adrenal, and oestrogen function. There were abnormalities of fatty acid metabolism in both. In 1 patient only continuous epidural anaesthesia completely relieved the pain.

Mella found only minimal changes in the fatty tumours histologically (Mella, 1967). She considered the 'lipomas' could be explained as an excessive amount of normal tissue. She thought the pain was due to pressure or stretching of nerves, or nerve trunks, by the fatty masses rather than neuromatous involvement in the tumour.

In family studies an inheritance pattern of autosomal dominance with variable expressivity appears to occur (Cantu et al., 1973).

One patient with Dercum's syndrome was given intravenous lignocaine $400 \mathrm{mg}$ daily (Iwane et al., 1976). This gave maximal pain relief after 20 minutes, and it persisted for approximately 10 hours.

Another group of disorders of importance to consider are those that effect the infrapatellar fat pad. This wedge-shaped pad occupies the pyramidal space between the femur, tibia, and patellar tendon. It is covered with synovial membrane and projects into the cavity, to change shape with every movement of the joint (Smillie, 1974a). It is effected by Hoffa's disease and what Smillie calls the 'premenstrual water-retention syndrome' (Smillie, 1974b).

Hoffa described what he considered to be a 'hyperplasia inflammatoria' of the normal intraarticular adipose tissue in the knee (Hoffa, 1904). He stated it could occur after even repeated slight trauma. The histology of the synovial fat pad reveals an extensive delicate network of fibrous strings interspersed with fat lobules the surface being covered with endothelial cells and small villi. Pain is felt on the medial side of the joint, and masses which are often larger than an egg may be palpated. Hoffa advocated surgical removal of the mass.

A woman who developed a radio-opaque mass after a fall on her knee 15 years previously has been described (Des Marchais and Gagnon, 1973). Although the result of operation in their patient is not given, these authors also advocate surgical removal.

The premenstrual water retention syndrome is characterised by pain in both knees, one usually greater than the other (Smillie, 1974b). The pain is made worse by progestogens and eased by wearing high heels. Reassurance is the only treatment required. The pathogenesis of this condition is attributed to the fact that premenstrually the swollen redundant fat pad becomes compressed during extension of the knee (limitation of knee extension by high heels probably explains their efficacy). The 
fat pad is supposed to have a considerable nerve plexus, and thus, with tension, pain results. The synovial membrane lining the fat pad is subject to compression, and with recurrence of the trauma at monthly intervals secondary inflammatory and fibrotic changes can occur.

We also examined 7 women with generalised massive obesity. None had localised fatty tissue masses. In addition we saw several moderately obese patients with localised fat hypertrophy around their knee joints in whom the fat was not tender. Thus in some patients the juxta-articular fatty masses may not be the cause of pain and a careful search for intra-articular or periarticular pathology should be made. In the experience of one of the authors (R.L.S.) osteoarthritis of the media 1tibiofemoral compartment and/or pes anserine bursitis was the predominant site of the discomfort. Local corticosteroid injections into the knee joint or pes anserine bursa in these cases (in contrast to our case 1) has proved to be of value in obtaining symptomatic relief. We thus conclude JAD is a real entity with a range of severity from what Smillie calls 'panniculitis' (Smillie, 1974c) to Dercum's syndrome (Kling, 1937). The importance of recognising this group of disorders is to distinguish painful fat pads near knees from other causes of knee pain such as osteoarthritis.

From the variety of treatments attempted it is apparent that none is completely satisfactory. In those patients whose primary disorder is JAD we recommend that an initial attempt be made to control symptoms with simple analgesics or nonsteroidal anti-inflammatory drugs and local heat or cold. Compression bandages can be tried. The role if any of continuous epidural anaesthesia (Blomstrand, et al. 1971) or daily intravenous lignocaine (Iwane et al., 1976) remains to be established.

It should be also noted that many of these patients have mild to moderate psychiatric illness such as depression or emotional lability, and this may contribute to their unsuccessful treatment, especially if secondary gain is involved.

\section{References}

Allen, E. V., and Hines, E. A. (1940). Lipedema of the legs: a syndrome characterised by fat legs and orthostatic edema. Proceedings of the Staff Meetings of the Mayo Clinic, 15, 184-187.
Blomstrand, R., Juhlin, L., Nordenstam, H., Ohlsson, R., Werner, B., and Engstrom, J. (1971). Adiposis dolorosa? associated with defects of lipid metabolism. Acta Dermatovenerologica, 51, 243-250.

Cantu, J. M., Ruiz-Barquin, E., Jimenez, M., Castillo, L., and Macotela-Ruiz, E. (1973). Autosomal dominant inheritance in adiposis dolorosa (Dercum's disease). $\frac{\bar{\rho}}{\bar{\phi}}$ Humangenetik, 18, 89-91.

Dercum, F. X. (1892). Three cases of a hitherto unclassified affection resembling in its grosser aspects obesity, butê associated with special nervous symptoms-adiposisdolorosa. American Journal of Medical Sciences, 104, 521-535.

Dercum, F. X., and McCarthy, D. J. (1902). Autopsy in a case of adiposis dolorosa. American Journal of Medicalo Sciences, 124, 994-1006.

Des Marchais, J., and Gagnon, P. A. (1973). Maladie d'Hoffaç (liposynovitis infrapatellaris, inflammation infrapatellar fat pad). Union Médicale du Canada, 102, 1313-1315.

Gram, H. C. (1930). A symptom triad of the post-climatic period (adipositas dolorosa-arthritis genuum-hyper- 6 tensio arterialis). Acta Medica Scandinavica, 73, 139-윽 207.

Hoffa, A. (1904). The influence of the adipose tissue with regard to the pathology of the knee joint. Journal of the American Medical Association, 43, 795-796.

Iwane, T., Maruyama, M., Matsuki, M., Ito, Y. and Shimoji, K. (1976). Management of intractable pain in adiposis dolorosa with intravenous administration of lidocaine. 6 Anesthesia and Analgesia, 55, 257-259.

Kling, D. H. (1937). Juxta-articular adiposis dolorosa. its significance and relation to Dercum's disease and osteo-O arthritis. Archives of Surgery, 34, 599-630.

Mella, B. A. (1967). Adiposis dolorosa. University of Michigan Medical Centre Journal, 33, 79-81.

Price, G. E. (1909). Adiposis dolorosa. American Journal of the Medical Sciences, 137, 705-715.

Smillie, I. S., (1974a). Lesions of the infra-patellar fat pad $\overrightarrow{\bar{\sigma}}$ and related synovial membrane diseases of the knee 3 joint. 1st Edn., pp. 151-152. Churchill-Livingstone: Edinburgh.

Smillie, I. S. (1974b). Lesions of the intra-patellar Fat Pad. and related Synovial Membrane Diseases of the Kneed Joint, 1st Edn., pp. 394-398. Churchill-Livingstone:응 Edinburgh.

Smillie, I. S. (1974c). Lesions of the intra-patellar Fat PadP. and related Synovial Membrane Diseases of the Knee Joint, 1st Edn., pp. 398-400. Churchill-Livingstone: Edinburgh.

Stallworth, J. M., Hennigar, G. R., Jonsson, H. T., and음 Rodriguez, O. (1974). The chronically swollen painful extremity. Journal of the American Medical Association, 0 228, 1656-1659.

Steiger, W. A., Litvin, H., Lasché, E. M., and Durant, T. M.N (1952). Adiposis dolorosa (Dercum's disease). New England Journal of Medicine, 247, 393-396.

Winkelman, N. W., and Eckel, J. L. (1925). Adiposis dolorosa N (Dercum's disease). Journal of the American Medical Association, 85, 1935-1939. 